\title{
Transcriptome differences between two sister desert poplar species under salt stress
}

\author{
Jian Zhang ${ }^{1}$, Jianju Feng ${ }^{1,2}$, Jing Lu', Yongzhi Yang ${ }^{1}$, Xu Zhang ${ }^{1}$, Dongshi Wan ${ }^{1}$ and Jianquan Liu*
}

\begin{abstract}
Background: Populus euphratica Oliv and P. pruinosa Schrenk (Salicaceae) both grow in dry desert areas with high summer temperatures. However, $P$. euphratica is distributed in dry deserts with deep underground water whereas $P$. pruinosa occurs in deserts in which there is underground water close to the surface. We therefore hypothesized that these two sister species may have evolved divergent regulatory and metabolic pathways during their interaction with different salt habitats and other stresses. To test this hypothesis, we compared transcriptomes from callus exposed to $24 \mathrm{~h}$ of salt stress and control callus samples from both species and identified differentially expressed genes (DEGs) and alternative splicing (AS) events that had occurred under salt stress.

Results: A total of 36,144 transcripts were identified and 1430 genes were found to be differentially expressed in at least one species in response to salt stress. Of these DEGs, 884 and 860 were identified in P. euphratica and P. pruinosa, respectively, while 314 DEGs were common to both species. On the basis of parametric analysis of gene set enrichment, GO enrichment in P. euphratica was found to be significantly different from that in P. pruinosa. Numerous genes involved in hormone biosynthesis, transporters and transcription factors showed clear differences between the two species in response to salt stress. We also identified 26,560 AS events which were mapped to 8380 poplar genomic loci from four libraries. GO enrichments for genes undergoing AS events in $P$. euphratica differed significantly from those in P. pruinosa.

Conclusions: A number of salt-responsive genes in both $P$. euphratica and $P$. pruinosa were identified and candidate genes with potential roles in the salinity adaptation were proposed. Transcriptome comparisons of two sister desert poplar species under salt stress suggest that these two species may have developed different genetic pathways in order to adapt to different desert salt habitats. The DEGs that were found to be common to both species under salt stress may be especially important for future genetic improvement of cultivated poplars or other crops through transgenic approaches in order to increase tolerance of saline soil conditions.
\end{abstract}

Keywords: P. euphratica, P. pruinosa, Salt tolerance, Salinity stress, Transcriptome, Differentially expressed genes, Alternative splicing

\section{Background}

Salinity and drought stresses are the two most important environmental factors limiting plant growth and development in semiarid and arid areas [1]. Over 100 countries in the world have been identified as being affected by salinity [2], and the scale of the problem seems to be increasing at an alarming rate [3]. Salinity, together with drought, has far-reaching implications for food security, economic sustainability and the irreplaceable biodiversity of any affected area, and it is anticipated that these

\footnotetext{
* Correspondence: liujq@nwipb.ac.cn

${ }^{1}$ State Key Laboratory of Grassland and Agro-Ecosystems, School of Life Sciences, Lanzhou University, Lanzhou 730000, Gansu, China

Full list of author information is available at the end of the article
}

challenges will be exacerbated by the projected impact of climate change. The effects of water-insufficiency stresses have been studied extensively; they limit water and micronutrient uptake and lead to closure of stomata, decline in carbon metabolism, stunted growth, ion/salt toxicity and reduced yield [3,4].

For plants to survive under such conditions, they must sense and respond to these abiotic stresses rapidly and in a complex manner [5], through signalling and regulatory pathways $[3,4,6]$ mediated by abscisic acid [7] or ethylene [8], generally resulting in altered expression of transcription factors [9], and in many cases in increased expression of genes encoding products required for osmoregulation, cell protection and/or acclimation [10-15]. These modifications

\section{Biomed Central}


may lead to changes in signal transduction, ionic homeostasis, scavenging of reactive oxygen species, accumulation of compatible solutes and growth regulation [3,6,16-18]. A common strategy for the identification of overall changes in gene expression under salt stress is to compare the transcriptomes of the targeted species or cultivars using microarrays and/or RNA-Seq technologies [19]. A plethora of comparisons between salt-sensitive and salt-tolerant cultivars of model and non-model plant species, including Arabidopsis [20-22], rice [23], poplar [24-27], tomato [28], potato [29], Medicago truncatula [30], sugarcane [31] and olive [32], have been reported to date. These studies have identified more than 30 families of transcription factors and numerous enzyme-encoding genes involved in responses to salt stress $[33,34]$. However, overall changes in gene expression and physiological responses to salt stress vary greatly between different species, particularly between sensitive and non-sensitive pairs of related species [35-39]. It is often difficult to ascertain whether these differences were caused by divergence during the course of evolution or were brought about through adaptive differentiation. It is therefore of interest to compare the overall changes in gene expression that occur in sister species under salt stress, as this will minimise phylogenetic effects.

Here we examine differences in the transcriptomes of two sister desert poplar species under salt stress. Populus serves as a model for elucidating physiological and molecular mechanisms of stress tolerance in tree species [40-42]. Both P. euphratica and P. pruinosa grow in dry deserts with high summer temperatures [43-46]. Both species can tolerate high salinity and survive $\mathrm{NaCl}$ concentrations of more than $300 \mathrm{mM}$ [47] in nutrient solution, and $P$. euphratica has been used as a model species for studying abiotic responses to salt or drought stress [27,48-50]. In addition to differences in leaf and hair morphology between the two species, they also occur in different types of habitat. P. euphratica is found in dry deserts with deep underground water while $P$. pruinosa is distributed in deserts where the underground water is closer to the surface, and therefore more accessible, but also saltier near ancient or extant rivers. It is likely that these two species have diverged due to ecological differentiation, in spite of ongoing gene flow [46].

In order to test whether regulatory and metabolic pathways in these two species have diverged during their adaptive interactions with salt and other stresses, the transcriptomes of callus subjected to $24 \mathrm{~h}$ of salt stress, and control callus samples, from $P$. euphratica and $P$. pruinosa were compared in order to identify differentially expressed genes (DEGs) and alternative splicing (AS) events that occurred in response to salt stress. Our results revealed that these two poplar species have both common and species-specific patterns of gene expression under salt stress. The dynamic transcriptome expression profiles of these sister species under salt stress obtained in this study may provide useful insights to inform further analyses of the mechanism of high salinity tolerance in plants. In addition, the genes found to be differentially expressed under salt stress in both species may facilitate the identification of key genes as potentially suitable targets for biotechnological manipulation with the aim of improving poplar salt tolerance.

\section{Results and discussion}

Analysis and mapping of Illumina-Solexa sequencing tags We used the Illumina-Solexa sequencing platform to sequence the $P$. euphratica [27] and P. pruinosa [51] transcriptomes obtained from the four treatments, including two unstressed callus samples as controls (P. euphratica control callus, PeuC; P. pruinosa control callus, PprC) and two salt-stressed callus samples as treatments (P. euphratica salt-stressed callus, PeuS; P. pruinosa salt-stressed callus, PprS). After removing low-quality sequences and trimming adapter sequences, $\sim 28$ million 75 -bp paired-end clean reads were generated from each of the cDNA libraries in the Illumina Genome Analyzer runs (Table 1). These tags from the four digital gene expression (DGE) libraries were mapped to the available $P$. trichocarpa transcript sequences. Approximately $80 \%$ of the tags had matches. Most (79.2-82.4\%) of the tags with matches were unique tags (matching only one poplar locus), while the remainder ( 17.6-20.8\%) were non-unique (matching more than one poplar locus) or unaligned. For more detailed investigation of gene expression in the different treatments, only unique tags were used in the analysis. In total, 36,144 transcripts were identified from the four conditions. The transcripts identified accounted for $80.3 \%$ of the 45,033 annotated genes in poplar. In both control and salt stress treatments, the numbers of mapped genes in $P$. euphratica (33,528 and 32,508 genes) were found to be similar to those in P. pruinosa (32,996 and 33,055 genes, respectively) (Table 1). We further compared the mapped genes among the four treatments (PeuC, PeuS, PprC and PprS), and found that $\sim 89.1 \%$ of them were present in at least two treatments (Figure 1).

\section{DEGs in the two species under salt stress}

To identify global transcriptional changes occurring under salt stress, we applied four independent metrics to identify genes that were differentially expressed between the 24-h salt-stressed callus and control callus samples in $P$. euphratica and P. pruinosa. For each metric, we selected those DEGs whose expression profiles met three criteria: (i) the FPKM value was $\geq 1$ in either of the libraries, (ii) $\log _{2}\left(\mathrm{FPKM}_{\text {salt }} / \mathrm{FPKM}_{\text {control }}\right)$ was $>1$ or $<-1$, and (iii) the adjusted $p$-value (FDR) was $<0.05$. In this study, DEGs with higher expression levels in salt-stressed callus when compared with control callus samples were termed 'up- 
Table 1 Summary of the Illumina-Solexa sequencing tags and their matches in the $P$. trichocarpa genome

\begin{tabular}{|c|c|c|c|c|c|}
\hline \multirow[t]{2}{*}{ Samples } & \multirow{2}{*}{$\begin{array}{l}\text { Matched } \\
\text { genes (\%) }\end{array}$} & \multicolumn{2}{|c|}{ Aligned tags } & \multirow{2}{*}{$\begin{array}{l}\text { Unaligned } \\
\text { tags (\%) }\end{array}$} & \multirow{2}{*}{$\begin{array}{c}\text { Total clear } \\
\text { tags }\end{array}$} \\
\hline & & Unique (\%) & Non-unique (\%) & & \\
\hline PeuC & $33,528(74.5)$ & $22,704,962(82.4)$ & $229,952(0.8)$ & $4,621,235(16.8)$ & $27,556,149$ \\
\hline PeuS & $32,508(72.2)$ & $23,375,397(80.6)$ & $297,806(1.0)$ & $5,314,269(18.4)$ & $28,987,472$ \\
\hline PprC & $32,996(73.3)$ & $22,298,805(79.2)$ & $241,822(0.9)$ & $5,599,162(19.9)$ & $28,139,789$ \\
\hline PprS & $33,055(73.4)$ & $23,027,167(81.0)$ & $364,836(1.3)$ & $5,046,367(17.7)$ & $28,438,370$ \\
\hline
\end{tabular}

PeuC, P. euphratica control callus; PeuS, P. euphratica salt-stressed callus; PprC, $P$. pruinosa control callus; PprS, $P$. pruinosa salt-stressed callus.

regulated', while those with lower expression levels in saltstressed callus were termed 'down-regulated'. There were 471 and 593 genes identified by all metrics as being upregulated in P. euphratica and P. pruinosa, respectively, and 413 and 267 genes identified by all metrics as downregulated in $P$. euphratica and $P$. pruinosa, respectively (Figure 2). There were more up-regulated DEGs in P. pruinosa than in P. euphratica, while there were more downregulated DEGs in P. euphratica than in P. pruinosa.

The DEGs identified were classified into eight clusters according to their expression patterns (Figure 3, Additional file 1). Of these eight clusters, four were up-regulated or down-regulated exclusively in a single species, as follows: up-regulated exclusively in P. euphratica (272 DEGs) or in P. pruinosa (394); down-regulated exclusively in P. euphratica (298) or in P. pruinosa (152). The remaining four clusters consisted of genes that were up- or down-regulated in the two species; two of these clusters showed similar co-regulation patterns whereas the other two showed opposing regulation patterns. In the two clusters with similar co-regulation patterns, 198 DEGs were co-up-regulated and 114 DEGs were co-downregulated in the two species. Within the co-up-regulated

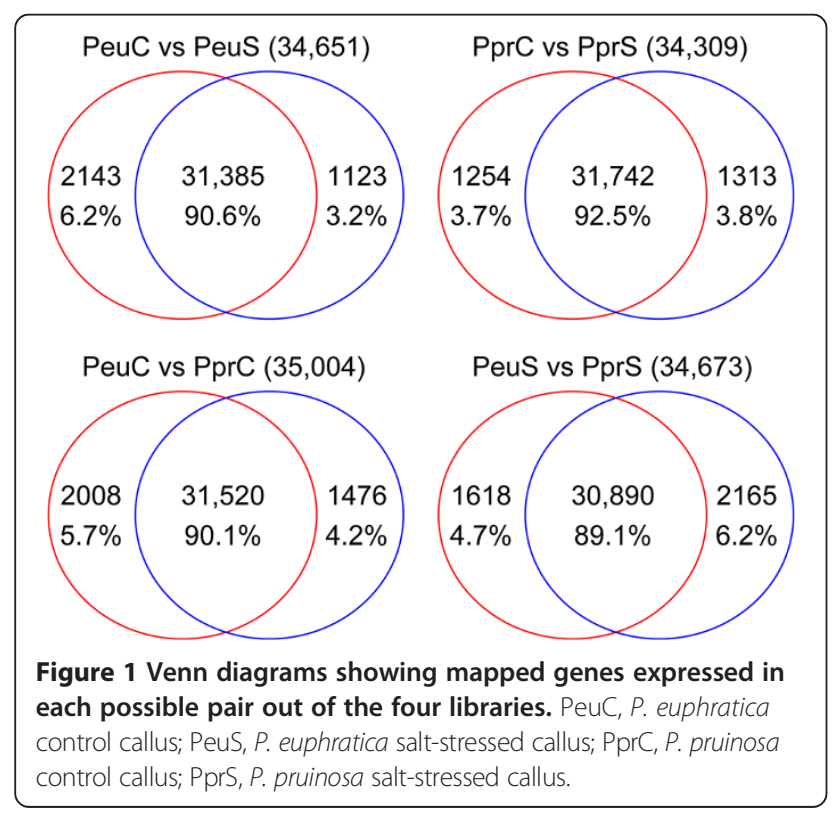

clusters, only one transcript (POPTR_0013s12880.1) was undetectable in the calli of the two species under unstressed conditions (Additional file 1), suggesting that this gene is expressed specifically under salt stress in both species. In the two clusters with opposing patterns of regulation, only 1 DEG was up-regulated in $P$. euphratica but down-regulated in P. pruinosa, and only 1 DEG was downregulated in $P$. euphratica but up-regulated in P. pruinosa. This result suggested that our integrated DEG identification was sensitive and reliable.

\section{Confirmation of differentially expressed candidate genes by qRT-PCR analysis}

To confirm the gene expression inferred from RNA-seq, a total of 21 candidate DEGs with salt-related process were selected for the qRT-PCR analyses, comprising 7 DEGs exclusively regulated in a single species, 8 co-upregulated and 6 co-down-regulated in the two species (Figure 4). Although the exact change did not exactly match each other, the expression trends of all 21 genes from qRT-PCR and Illumina-Solexa RNA sequencing analyses were largely consistent (Pearson's correlation coefficient $r=0.8$ ), demonstrating the reliability of the RNA-seq results (Figure 4).

Gene functional categories of two species under salt stress Firstly, an overview of the main results was obtained by WEGO and the DEGs were assigned to GO terms in the three component ontologies (Figure 5). Then, groups of genes with functions involved in salt responses were identified using parametric analysis of gene set enrichment (PAGE) (Table 2). GO enrichment in P. euphratica was significantly different from that in $P$. pruinosa. In the Cellular Component ontology, 'apoplast' (GO:0044464) appeared to respond to salt stress in both species; while 'cell part' (GO:0044464) and 'cell' (GO:0005623) were enriched only in $P$. euphratica; whereas 'extracellular region' (GO:0005576), 'external encapsulating structure' (GO:0030312) and 'cell wall' (GO:0005618) were enriched only in P. pruinosa. In the Molecular Function ontology, 'cofactor binding' (GO:0048037), 'coenzyme binding' (GO:0050662), 'peptidase inhibitor activity' (GO:0030414) and 'endopeptidase inhibitor activity' (GO:0004866) were enriched in both species, while another nine terms from 

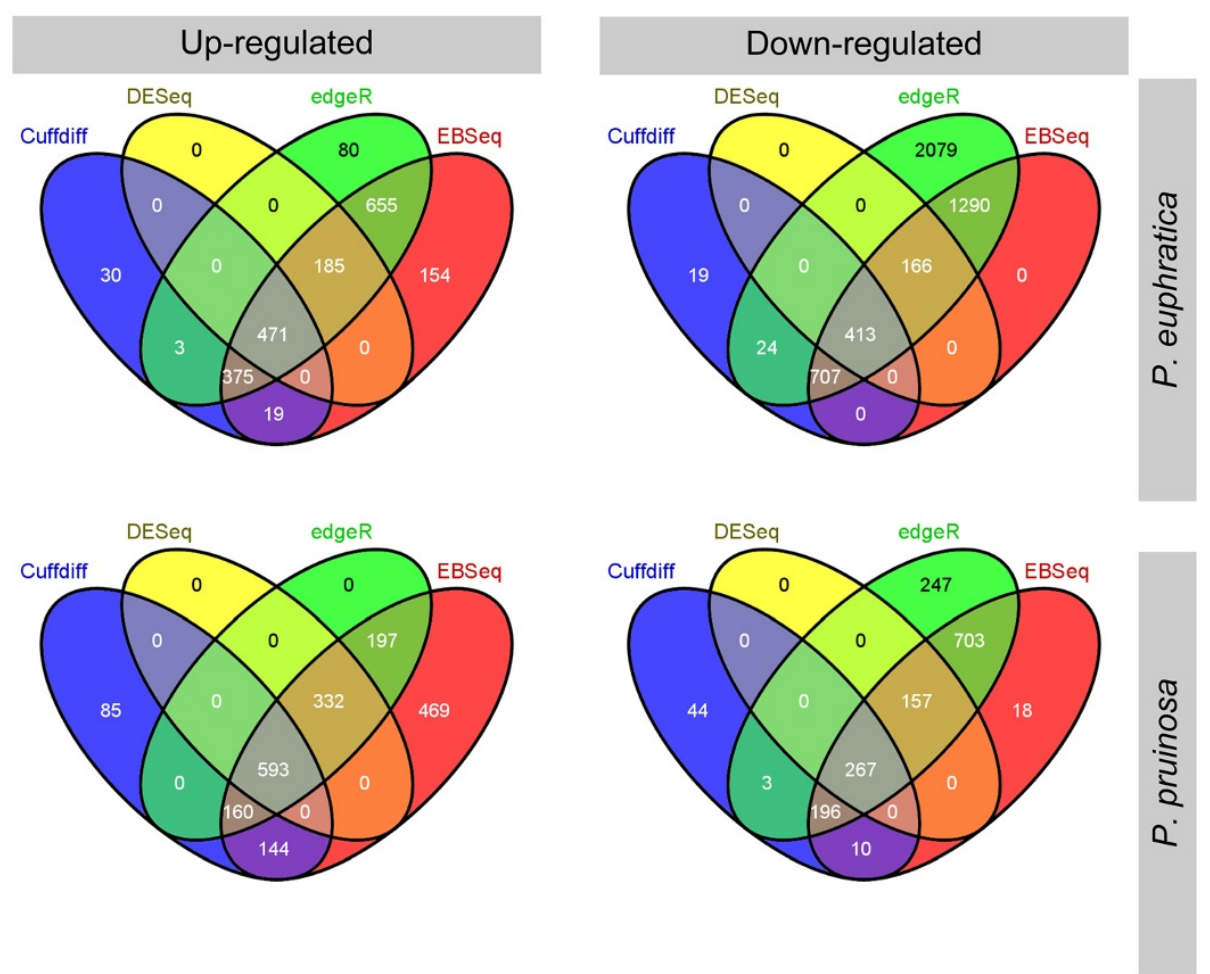

Figure 2 Comparison of four metrics for classifying DEGs. Venn diagrams of the numbers of up-regulated (left) and down-regulated (right) genes identified by four comparisons of control callus and salt-stressed callus from P. euphratica (top) and P. pruinosa (bottom).

the Molecular Function ontology were enriched exclusively in P. euphratica. Six terms from the Biological Processes ontology were enriched exclusively in $P$. euphratica and three terms were enriched in both species. The GO terms enriched in P. euphratica were related to responses to stress and metabolic processes, and the most highly enriched term was 'response to stress' (GO:0006950). We also used singular enrichment analysis (SEA) to identify functional groups of genes differentially expressed in the two species under salinity (Additional file 2). GO enrichment for genes

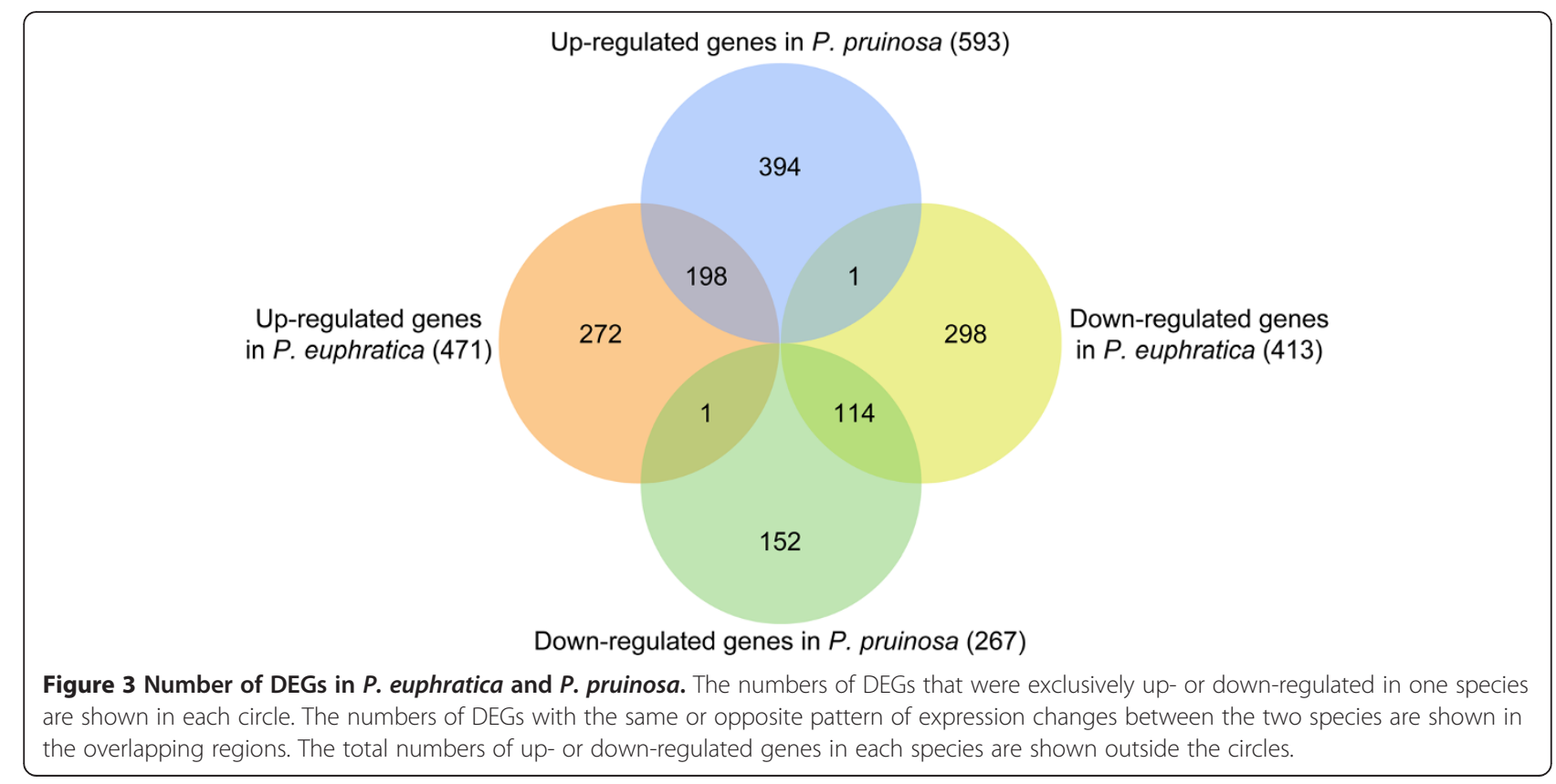




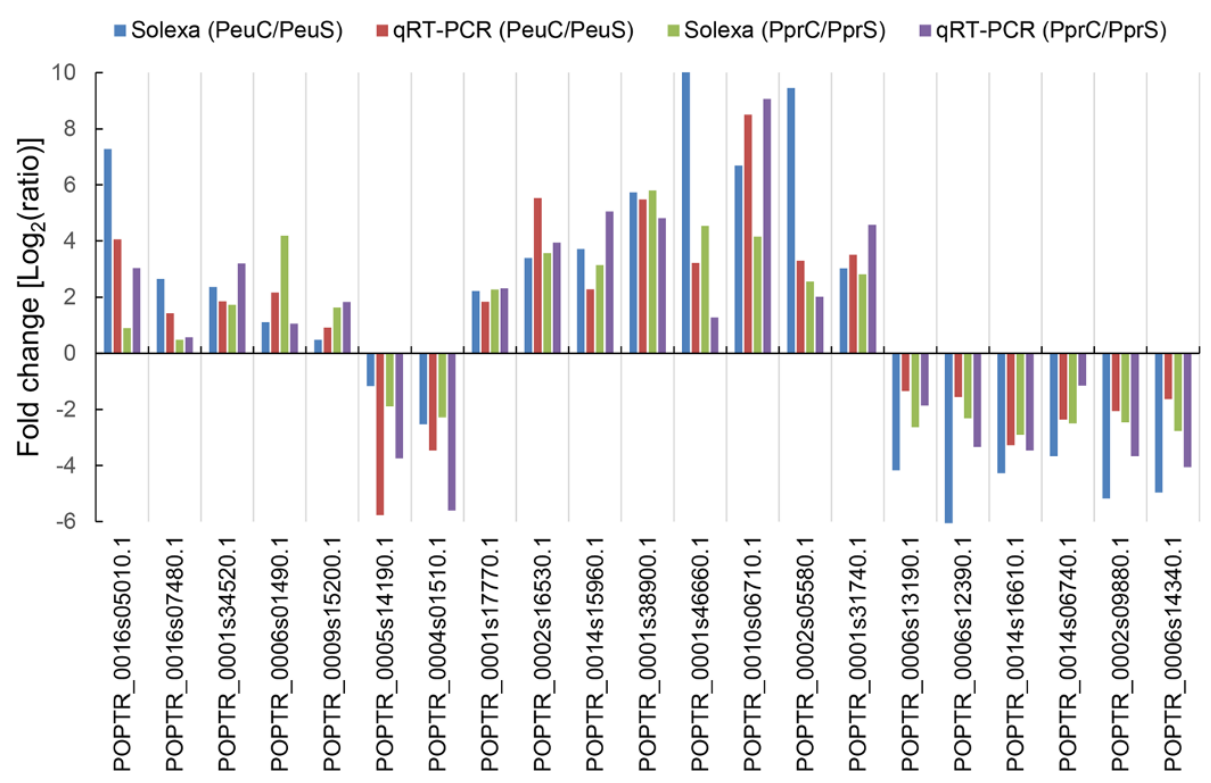

Figure 4 Expression pattern validation of selected genes by qRT-PCR. Expression changes of 21 DEGs in the salt-stressed calli relative to the control calli were measured by qRT-PCR. The transcriptional level of candidate genes was examined by real time PCR with three biological replications and actin was used as an internal control. Results were present as target/reference ratios normalized by the calibrator. No significant differences were shown between qRT-PCR and the Illumina data (Pearson's correlation coefficient $r=0.8$ ). The $Y$-axis indicates the fold change of transcript abundance in salt-stressed callus relative to the control callus. PeuC, P. euphratica control calli; PeuS, P. euphratica salt-stressed calli; PprC, P. pruinosa control calli; PprS, P. pruinosa salt-stressed calli.

up-regulated or down-regulated exclusively in P. euphratica was significantly different from that in $P$. pruinosa. The detected differences suggested that these two desert poplars might have developed different genetic pathways for adaptation to differentiated salty desert habitats.

\section{Differences in expression of hormone-related genes in} the two species under salt stress

Using the Kyoto Encyclopedia of Genes and Genomes (KEGG) database as our source of annotations, 583 out of 803 Populus genes annotated as being involved in

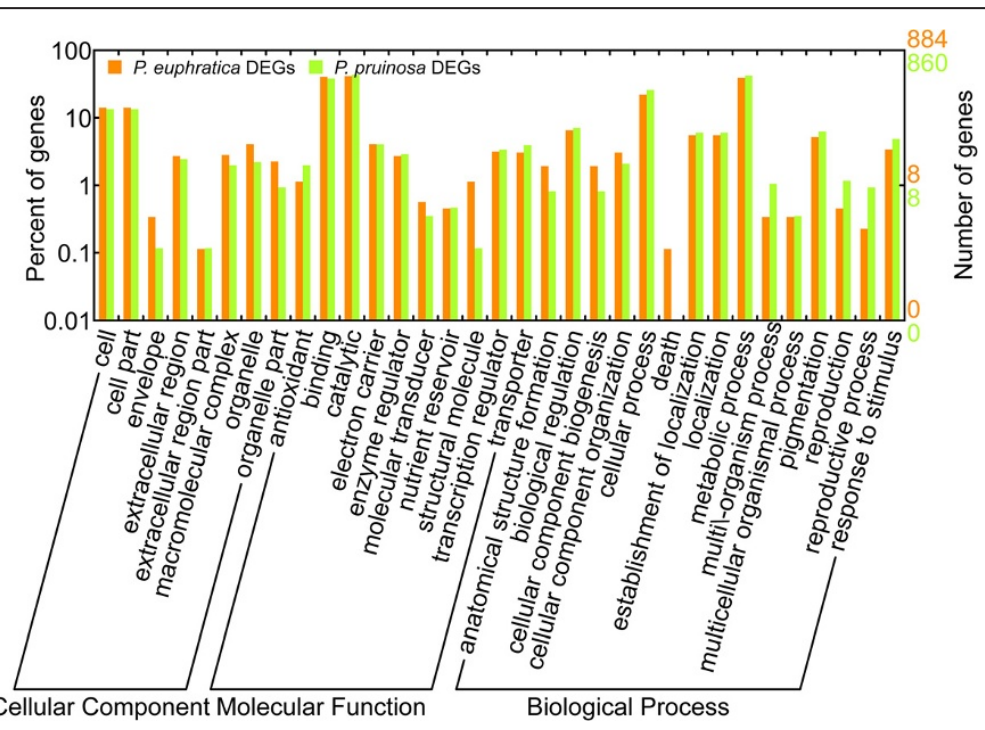

Figure 5 Gene ontology (GO) annotation of salt-responsive genes compared between $P$. euphratica and $P$. pruinosa. WEGO was Used to produce the graph. We divided the sets into the three major GO domains: biological process, cellular component and molecular function, and the number (right $y$-axis) and percentage (left y-axis) of genes were calculated. 
Table 2 Gene ontology (GO) enrichment analyses for salt-responsive genes compared between $P$. euphratica and P. pruinosa ${ }^{1}$

\begin{tabular}{|c|c|c|c|c|c|c|c|c|c|}
\hline \multirow[t]{2}{*}{ GO term } & \multirow[t]{2}{*}{ Onto $^{2}$} & \multirow[t]{2}{*}{ Description } & \multirow[t]{2}{*}{ Number } & \multicolumn{3}{|c|}{ P. euphratica } & \multicolumn{3}{|c|}{ P. pruinosa } \\
\hline & & & & Z-score & Mean $^{3}$ & FDR & Z-score & Mean & FDR \\
\hline GO:0006950 & $P$ & Response to stress & 50 & 7.2 & 5.1 & $4.50 \mathrm{e}-11$ & 3.1 & 1.8 & 0.21 \\
\hline GO:0050896 & $P$ & Response to stimulus & 54 & 6.7 & 4.6 & $1.60 \mathrm{e}-09$ & 3.1 & 1.8 & 0.21 \\
\hline GO:0006952 & $P$ & Defense response & 16 & 6.4 & 7.7 & $1.50 \mathrm{e}-08$ & 3.2 & 2.9 & 0.11 \\
\hline GO:0009607 & $P$ & Response to biotic stimulus & 15 & 5.9 & 7.4 & 4.20e-07 & 3.4 & 3.1 & 0.063 \\
\hline GO:0009308 & $P$ & Amine metabolic process & 22 & 3.5 & 3.7 & 0.047 & 2.3 & 2 & 1 \\
\hline GO:0044264 & $P$ & Cellular polysaccharide metabolic process & 13 & -3.6 & -4.3 & 0.036 & -4.6 & -3 & 0.00034 \\
\hline GO:0044042 & $\mathrm{P}$ & Glucan metabolic process & 13 & -3.6 & -4.3 & 0.036 & -4.6 & -3 & 0.00034 \\
\hline GO:0006073 & $P$ & Cellular glucan metabolic process & 13 & -3.6 & -4.3 & 0.036 & -4.6 & -3 & 0.00034 \\
\hline GO:0044260 & $P$ & Cellular macromolecule metabolic process & 159 & -3.6 & -1 & 0.035 & -2.2 & 0.11 & 1 \\
\hline GO:0048037 & $\mathrm{F}$ & Cofactor binding & 67 & 8.1 & 4.9 & $3.90 \mathrm{e}-14$ & 3.8 & 1.9 & 0.011 \\
\hline GO:0050662 & $\mathrm{F}$ & Coenzyme binding & 53 & 7.9 & 5.4 & $2.00 e-13$ & 3.5 & 2 & 0.034 \\
\hline GO:0030414 & $\mathrm{F}$ & Peptidase inhibitor activity & 21 & 7.9 & 8.3 & $2.50 \mathrm{e}-13$ & 4.3 & 3.2 & 0.0015 \\
\hline GO:0004866 & $\mathrm{F}$ & Endopeptidase inhibitor activity & 21 & 7.9 & 8.3 & $2.50 \mathrm{e}-13$ & 4.3 & 3.2 & 0.0015 \\
\hline GO:0050660 & $\mathrm{F}$ & FAD binding & 33 & 7.4 & 6.3 & $1.40 \mathrm{e}-11$ & 2.9 & 2 & 0.37 \\
\hline GO:0016491 & $\mathrm{F}$ & Oxidoreductase activity & 191 & 6.6 & 2.5 & $2.60 \mathrm{e}-09$ & 2.3 & 1.1 & 1 \\
\hline GO:0004857 & $\mathrm{F}$ & Enzyme inhibitor activity & 32 & 6.5 & 5.7 & $6.50 \mathrm{e}-09$ & 3.4 & 2.3 & 0.066 \\
\hline GO:0030234 & $\mathrm{F}$ & Enzyme regulator activity & 33 & 6.2 & 5.4 & $3.80 \mathrm{e}-08$ & 3.2 & 2.1 & 0.13 \\
\hline GO:0030246 & $\mathrm{F}$ & Carbohydrate binding & 24 & 4.5 & 4.6 & 0.00052 & 2.3 & 1.9 & 1 \\
\hline GO:0004497 & $\mathrm{F}$ & Monooxygenase activity & 42 & 4 & 3.2 & 0.0052 & 1.1 & 1.1 & 1 \\
\hline GO:0003824 & $\mathrm{F}$ & Catalytic activity & 504 & 3.7 & 1 & 0.019 & 0.93 & 0.72 & 1 \\
\hline GO:0046906 & $\mathrm{F}$ & Tetrapyrrole binding & 64 & 3.5 & 2.3 & 0.042 & 0.12 & 0.65 & 1 \\
\hline GO:0020037 & $\mathrm{F}$ & Heme binding & 64 & 3.5 & 2.3 & 0.042 & 0.12 & 0.65 & 1 \\
\hline GO:0017171 & $\mathrm{F}$ & Serine hydrolase activity & 13 & -3.4 & -4.1 & 0.059 & -3.5 & -2.1 & 0.036 \\
\hline GO:0008236 & $\mathrm{F}$ & Serine-type peptidase activity & 13 & -3.4 & -4.1 & 0.059 & -3.5 & -2.1 & 0.036 \\
\hline GO:0016758 & $\mathrm{F}$ & Transferase activity, transferring hexosyl groups & 16 & -3.1 & -3.3 & 0.2 & -3.8 & -2.1 & 0.011 \\
\hline GO:0016762 & $\mathrm{F}$ & Xyloglucan:xyloglucosyl transferase activity & 10 & -3.1 & -4.2 & 0.2 & -4.2 & -3.1 & 0.0023 \\
\hline GO:0048046 & C & Apoplast & 10 & -3.1 & -4.2 & 0.047 & -4.2 & -3.1 & 0.00054 \\
\hline GO:0044464 & C & Cell part & 169 & -3.2 & -0.87 & 0.03 & -1.1 & 0.36 & 1 \\
\hline GO:0005623 & C & Cell & 169 & -3.2 & -0.87 & 0.03 & -1.1 & 0.36 & 1 \\
\hline GO:0005576 & C & Extracellular region & 12 & -2.4 & -3 & 0.33 & -3.3 & -2.1 & 0.02 \\
\hline GO:0030312 & C & External encapsulating structure & 18 & -2.8 & -2.8 & 0.11 & -3.8 & -1.9 & 0.0033 \\
\hline GO:0005618 & C & Cell wall & 18 & -2.8 & -2.8 & 0.11 & -3.8 & -1.9 & 0.0033 \\
\hline
\end{tabular}

${ }^{1}$ The 1430 DEGs were analyzed by parametric analysis of gene set enrichment (PAGE).

${ }^{2}$ Onto, Gene Ontology domain; P, biological process; $F$, molecular function; $C$, cellular component.

${ }^{3}$ Mean, mean $\log _{2}$ expression ratio, $>0$ represents up-regulation, $<0$ represents down-regulation.

hormone biosynthesis [52] were detected in the four libraries and 59 of these genes were differentially expressed in either $P$. euphratica or $P$. pruinosa under salt stress (Additional file 3). Among these hormone biosynthesisrelated DEGs, 37 were identified in P. euphratica, of which 36 were up-regulated and one was down-regulated during salt stress, while 39 were identified in $P$. pruinosa, including 36 up-regulated and 3 down-regulated DEGs; only 17 were co-regulated in the two species. Hierarchical clustering of the 59 hormone-related DEGs showed overall differences between $P$. euphratica and $P$. pruinosa in response to salt stress (Figure 6). Under salt stress, most hormone-related DEGs were co-up-regulated or co-down-regulated in both species. Interestingly, three ABA metabolism-related genes (ABA1, POPTR_0007s10980.1; and two genes encoding 9-cis-epoxycarotenoid dioxygenase (NCEDs), POPTR 0011s11370.1 and POPTR_0001s40420.1) were up-regulated exclusively in $P$. pruinosa. 


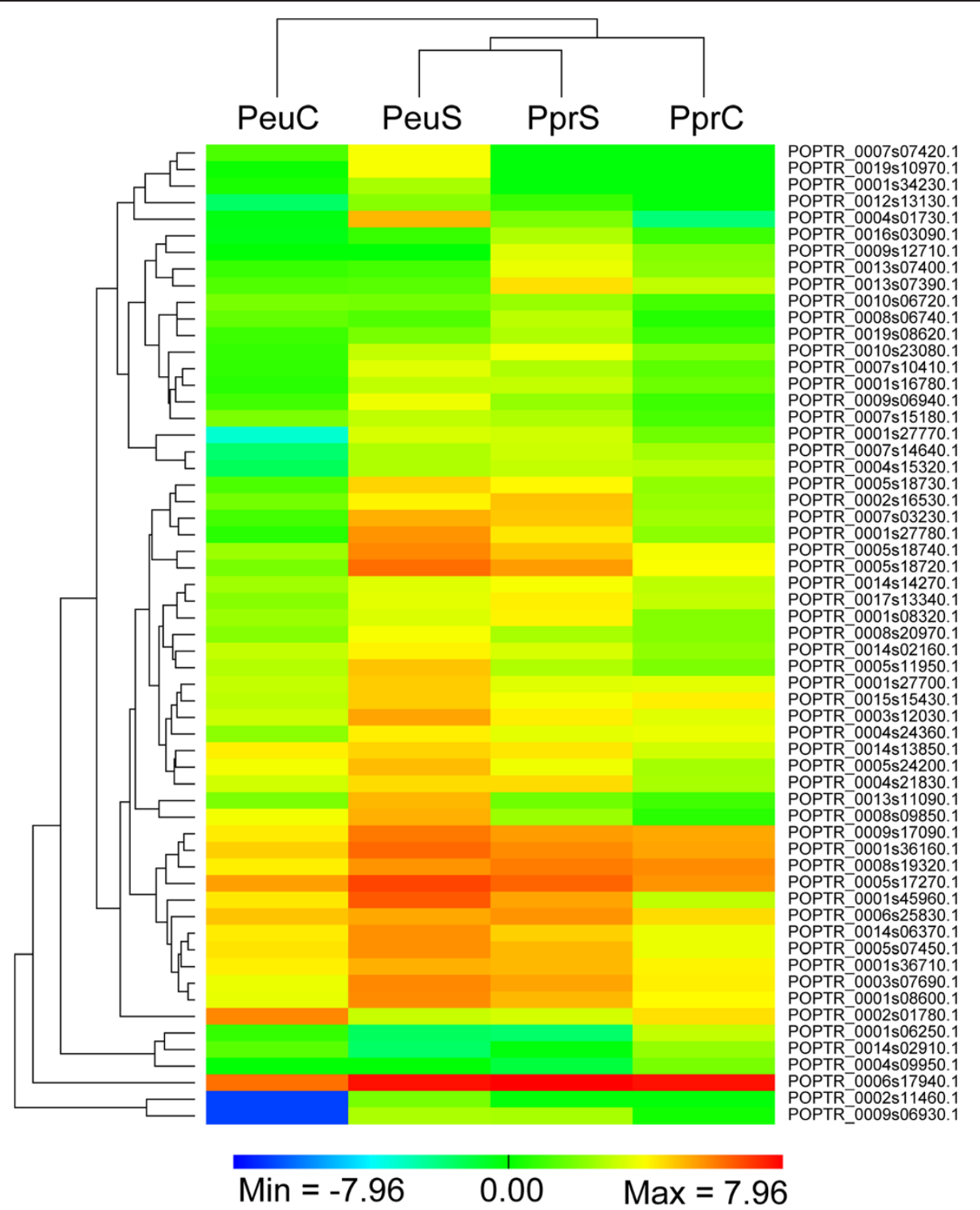

Figure 6 Hierarchical clustering of $\mathbf{5 8}$ genes related to plant hormone biosynthesis. Hierarchical cluster analysis was conducted using the software PermutMatrix v1.9.3 and displayed as a log-(FPKM) value for the intensity of expression of each DEG. FPKM, number of fragments per kilobase of exon per million fragments mapped; PeuC, P. euphratica control callus; PeuS, P. euphratica salt-stressed callus; PprC, P. pruinosa control callus; PprS, P. pruinosa salt-stressed callus.

\section{Differences in expression of transporter-encoding genes in the two species under salt stress}

On the basis of annotations in the database of Arabidopsis thaliana transporter proteins (http://www.membranetransport.org/all_type_btab.php?oOID=atha1), a total of 99 genes differentially regulated according to all metrics in either $P$. euphratica or $P$. pruinosa during salt stress were categorized as transporters (Additional file 4). Among these, 49 DEGs were identified in P. euphratica, of which 25 were up-regulated and 24 down-regulated during salt stress, while 66 were differentially regulated in $P$. pruinosa, consisting of 51 up-regulated and 14 down-regulated DEGs, and 16 were co-regulated in the two species. For example, we found that POPTR 0003s13470.1, POPTR_0008s14670.1 and POPTR_0006 s11590.1, which are homologous to Arabidopsis thaliana $\mathrm{Na}^{+} / \mathrm{H}^{+}$antiporter 18 (AT5G41610, CHX18), potassium transporter 6 (AT1G70300, KUP6) and ABC transporter (AT1G66950, ABCG39), respectively, were co-up-regulated in both species. However, POPTR_ 0005s04660.1 and POPTR_0014s12700.1, which are homologous to Arabidopsis thaliana sodium/hydrogen exchanger 2 (AT3G05030, NHX2) and potassium transporter 5 (AT4G13420, HAK5) genes, were up- 
regulated only in $P$. euphratica. In contrast, POPTR 0004s23680.1 and POPTR_0013s08110.1, which are homologous to Arabidopsis thaliana chloride channel protein CLC-c (AT5G49890, CLC-C) and potassium transporter 2 (AT2G40540, KT2) genes respectively, were up-regulated exclusively in $P$. pruinosa. These results corroborate previous findings [27,53-55], and confirm that genes encoding proteins such as sodium and potassium ion transmembrane transporters, and chloride channel and $\mathrm{ABC}$ transporters, which are important for maintaining and re-establishing homeostasis in the cytoplasm, are induced to high levels in response to salinity stress [16].

\section{Differences in expression of transcription factor genes in the two species under salt stress}

We identified 4016 transcription factors in Populus trichocarpa and classified them into 92 families (Additional file 5) based on published annotations. A total of 115 genes that were differentially regulated in either $P$. euphratica or $P$. pruinosa during salt stress were categorized as transcription factors (Additional file 6). Of these, 59 DEGs were identified in $P$. euphratica, including 24 that were upregulated and 35 down-regulated during salt stress, while 73 DEGs were identified in P. pruinosa, of which 52 were up-regulated and 21 down-regulated. Only 17 DEGs were co-regulated in both species (Table 3, Additional file 6). Several of the transcription factors, such as AP2/ERF and bZIP, which are known to be induced by stress in model plant species (Arabidopsis thaliana and rice) $[56,57]$, were highly expressed in response to salinity stress in P. euphratica or P. pruinosa.

Table 3 Transcription factors differentially expressed in the two species under salinity stress

\begin{tabular}{ccc}
\hline Transcription factor family & P. euphratica & P. pruinosa \\
\hline AP2/ERF & 13 & 11 \\
bZIP & 1 & 2 \\
MYB & 3 & 9 \\
WRKY & 5 & 8 \\
NAC & 7 & 8 \\
C2H2 & 2 & 2 \\
bHLH & 3 & 0 \\
MYB-like & 1 & 2 \\
GARP-G2-like & 1 & 0 \\
HB, Homeobox & 2 & 2 \\
GRAS & 2 & 2 \\
Other & 19 & 27 \\
Total & 59 & 73 \\
\hline
\end{tabular}

The co-up-regulated DEGs in the two species under salt stress and allele mining

A total of 198 co-up-regulated DEGs in the two species were identified in salt stress (Additional file 1) and the important ones were selected and listed in Table 4. The candidate genes identified in the present study contained both the previously reported salt-responsive genes and some species-specific ones. Of them, most genes were involved in and highly enriched in functional categories such as response to stress, signal transduction, transmembrane transport, transcriptional regulation and basic metabolic processes (Additional files 1 and 2). These findings are beneficial to allele mining of two poplar species related to their common or differentiated response to stressed habitats in the future. Allele mining based on the candidate genes were found to be important in dissecting naturally selected allelic variations that controlled differentiated traits $[58,59]$. In addition, promoters are found to play a key role in gene regulation, and any change in these regions will change gene expression and the controlled traits. Therefore, the identified variations through such an approach may be mainly located in the promoter regions [60]. Overall, the co-up-regulated DEGs identified in the present study provide critical genetic bases for further allele mining, functional analyses and transgenic practices for developing the salt-tolerant poplars and crops.

A comparison of DEGs identified by our results and other transcriptome studies of the salt-stressed poplars

In order to test the consistency of DEGs across different treatments and approaches, we compared DEGs between our results and other available transcriptome studies of the salt stressed poplars. Ottow et al. [48] examined changes in transcript levels of various genes known to be involved in salt or general stress signaling or adaptation in $P$. euphratica leaves by dot-blot expression. They identified nine genes with significant changes in response to salt stress. Some of them were be confirmed in the present study, for example, galactinol synthase 2 (GolS2, POPTR_ 0013s00730.1), calcineurin B-like protein 4 (CBL 4, POPTR_0015s01550.1), alternative oxidase 1A (POPTR_ 0012s01630.1) and 1-aminocyclopropane-1-carboxylate oxidase (POPTR_0011s00970.1) (Additional file 1). Galactinol synthase (GolS) catalyzes the first step in the biosynthetic pathway of raffinose oligosaccharides using galactose and myo-inositol as substrates and this gene was also up-regulated in plants under cold, heat, drought, and salt stress [21,61,62]. Significant increases in galactinol synthase and alternative oxidase after salt stress point to shifts in carbohydrate metabolism and suppression of reactive oxygen species in mitochondria under salt stress [48]. In addition, $\mathrm{Gu}$ et al. [63] identified 54 genes with altered transcript accumulation in the salt-stressed $P$. euphratica by microarray hybridization. The genes of 
Table 4 List of co-up-regulated DEGs in the two species under salinity stress

\begin{tabular}{|c|c|c|c|c|c|c|c|c|}
\hline \multirow[t]{2}{*}{ Transcript name } & \multirow[t]{2}{*}{ Arabidopsis } & \multirow[t]{2}{*}{ Gene annotation } & \multicolumn{4}{|c|}{ FPKM } & \multirow{2}{*}{$\begin{array}{l}\text { log2 Ratio } \\
\text { (PeuS/PeuC) }\end{array}$} & \multirow{2}{*}{$\begin{array}{l}\text { log2 Ratio } \\
\text { (PprS/PprC }\end{array}$} \\
\hline & & & PeuC & PeuS & PprC & PprS & & \\
\hline POPTR_0008s17940.1 & AT3G04120 & glyceraldehyde-3-phosphate dehydrogenase & 17.64 & 430.78 & 19.83 & 321.82 & 4.61 & 4.02 \\
\hline POPTR_0006s25280.1 & AT5G25880 & malate dehydrogenase (NADP+) & 48.70 & 190.54 & 28.89 & 85.17 & 1.97 & 1.56 \\
\hline POPTR_0007s14250.1 & AT4G37870 & phosphoenolpyruvate carboxykinase [ATP] & 82.37 & 565.15 & 92.96 & 253.92 & 2.78 & 1.45 \\
\hline POPTR_0006s11590.1 & AT1G66950 & ABC transporter $\mathrm{G}$ family member 39 & 3.02 & 36.26 & 5.99 & 17.88 & 3.59 & 1.58 \\
\hline POPTR_0001s26210.1 & AT4G16260 & catalytic/cation binding/hydrolase & 0.05 & 132.37 & 0.00 & 15.30 & 11.41 & 13.90 \\
\hline POPTR_0015s05290.1 & AT1G73480 & alpha/beta-hydrolase domain-containing protein & 1.90 & 17.84 & 0.63 & 10.20 & 3.23 & 4.02 \\
\hline POPTR_0016s01570.1 & AT3G21760 & UDP-glycosyltransferase-like protein & 2.87 & 20.08 & 2.06 & 13.49 & 2.81 & 2.71 \\
\hline POPTR_0006s12220.1 & AT3G53150 & UDP-glucosyl transferase 73D1 & 1.27 & 20.29 & 1.56 & 18.09 & 4.00 & 3.54 \\
\hline POPTR_0005s21690.1 & AT2G33710 & ethylene-responsive transcription factor ERF112 & 0.93 & 27.49 & 1.18 & 23.49 & 4.89 & 4.32 \\
\hline POPTR_0002s04020.1 & AT3G23240 & ethylene-responsive transcription factor $1 \mathrm{~B}$ & 1.44 & 28.29 & 3.29 & 14.91 & 4.29 & 2.18 \\
\hline POPTR_0008s14670.1 & AT1G70300 & Potassium transporter 6 & 35.29 & 191.46 & 55.07 & 133.58 & 2.44 & 1.28 \\
\hline POPTR_0010s09370.1 & AT3G22740 & homocysteine S-methyltransferase 3 & 0.13 & 16.64 & 0.31 & 12.10 & 7.04 & 5.28 \\
\hline POPTR_0015s11130.1 & AT5G13080 & putative WRKY transcription factor 75 & 24.38 & 143.60 & 30.56 & 286.38 & 2.56 & 3.23 \\
\hline POPTR_0010s10010.1 & AT5G26340 & sugar transport protein 13 & 5.48 & 116.42 & 8.20 & 72.52 & 4.41 & 3.15 \\
\hline POPTR_0001s09000.1 & AT4G1 1650 & osmotin-like protein OSM34 & 0.07 & 245.80 & 1.04 & 165.57 & 11.83 & 7.32 \\
\hline POPTR_0004s22170.1 & AT5G60700 & glycosyltransferase family protein 2 & 0.09 & 4.58 & 0.19 & 3.96 & 5.68 & 4.40 \\
\hline POPTR_0009s15100.1 & AT1G08250 & arogenate dehydratase 6 & 37.86 & 158.76 & 31.10 & 154.12 & 2.07 & 2.31 \\
\hline POPTR_0017s04590.1 & AT5G53970 & tyrosine aminotransferase & 29.34 & 253.35 & 24.46 & 201.65 & 3.11 & 3.04 \\
\hline POPTR_0006s16610.1 & AT4G28940 & Phosphorylase-like protein protein & 6.75 & 153.46 & 4.72 & 113.79 & 4.51 & 4.59 \\
\hline
\end{tabular}

them, responsible for hydroxyproline-rich glycoprotein, carbonic anhydrase 2, cytochrome P450, aquaporin, sucrose synthase and aspartate aminotransferase were confirmed in these present study. These genes were also revealed to be salt-responsive in other studies [26,27,64].

The drought responses of plants are similar to those in response to salinity because both stresses lead to physiological water deficit [65]. Bogeat-Triboulot et al. [66] provided a comprehensive analysis of $P$. euphratica subjected to gradual soil water depletion, and observed 110 regulatory and protective genes involved in long-term response to drought. Similar results were also found by Cohen et al. [25] and Tang et al. [67]. Among them, those genes involved in metabolites of proline, raffinose, galactose, inositol and sucrose under drought stress were found to have changed their expressions in response to salt stress in the presnet study. An increase in galactinol, raffinose and stachyose content may have improved osmoprotection and ROS scavenging when poplars were stressed by drought or salt. However, in the present study, we identified numerous more transcripts with significant up-regulations in both poplars when stressed, including UDP-glycosyltransferase-like protein, FADbinding and BBE domain-containing protein, putative nucleoredoxin 1, and glyceraldehyde-3-phosphate dehydrogenase. All these newly identified genes should have also played an important role during salt adaptation of two species. Their functions and molecular mechanisms need further clarifications in the future.

\section{Alternative splicing of transcripts in the two species under salt stress}

Finally, to investigate the role of alternative splicing (AS) in response to salt stress, we conducted a survey of transcript isoforms across the four libraries and examined six common types of 'alternative splicing events'. For each of these event types, reads deriving from specific regions can be used to identify the expression of one alternative isoform or the other (Table 5). We identified 26,560 AS events which were mapped to the 8380 poplar genomic loci from the four libraries, suggesting that $\sim 20 \%$ of 40,668 loci in poplar are potentially subject to AS. This observed AS percentage is comparable to the percentage of the genes shown to undergo AS in $A$. thaliana (21.8\%) and rice (21.2\%) [68]. In both control and salt stress treatments, the number of genes exhibiting AS events in P. euphratica (6662 and 5850 genes) is similar to that in P. pruinosa (6192 and 5765 genes), respectively. In addition to the AS loci (4115) common to both species, around 346 and 243 of the loci show AS events only in either $P$. euphratica or P. pruinosa in response to salt stress (Figure 7). We further classified those genes that underwent AS events on the basis of functional ontology. GO enrichment for the genes 
Table 5 Alternative splicing events in response to salt stress in $P$. euphratica and $P$. pruinosa

\begin{tabular}{lccccc}
\hline Type of event & PeuC & PeuS & PprC & PprS & Total \\
\hline Skipped exon & 13,323 & 12,130 & 11,655 & 10,749 & 20,334 \\
Retained intron & 2867 & 2195 & 2182 & 1776 & 4795 \\
Alternative 5' splice site (A5SS) & 274 & 294 & 282 & 269 & 529 \\
Alternative 3' splice site (A3SS) & 250 & 232 & 251 & 230 & 430 \\
Alternative first exon (AFE) & 162 & 129 & 148 & 142 & 222 \\
Alternative last exon (ALE) & 157 & 145 & 154 & 144 & 250 \\
Total AS events & 17,033 & 15,125 & 14,672 & 13,310 & 26,560 \\
Loci having AS events & 6662 & 5850 & 6192 & 5765 & 8380 \\
\hline
\end{tabular}

PeuC, P. euphratica control callus; PeuS, P. euphratica salt-stressed callus; PprC, $P$. pruinosa control callus; PprS, $P$. pruinosa salt-stressed callus.

displaying AS events exclusively in P. euphratica was significantly different from that for genes undergoing AS in P. pruinosa (Additional file 7).

\section{Conclusions}

Our transcriptional profiling analysis revealed numerous genes that were differentially expressed in both $P$. euphratica and $P$. pruinosa under salt stress. The differential expressions of the selected genes inferred from RNA-seq were confirmed by qRT-PCR data. Gene ontology analyses of these DEGs suggested that GO enrichment in $P$. euphratica was significantly different from that in P. pruinosa. We found that numerous genes involved in hormone biosynthesis, or encoding transporters or transcription factors, showed different expression patterns between these two species under salt stress. These differences suggest that these two desert poplars may have developed species-specific pathways for adaptation to salinity during the course of ecological

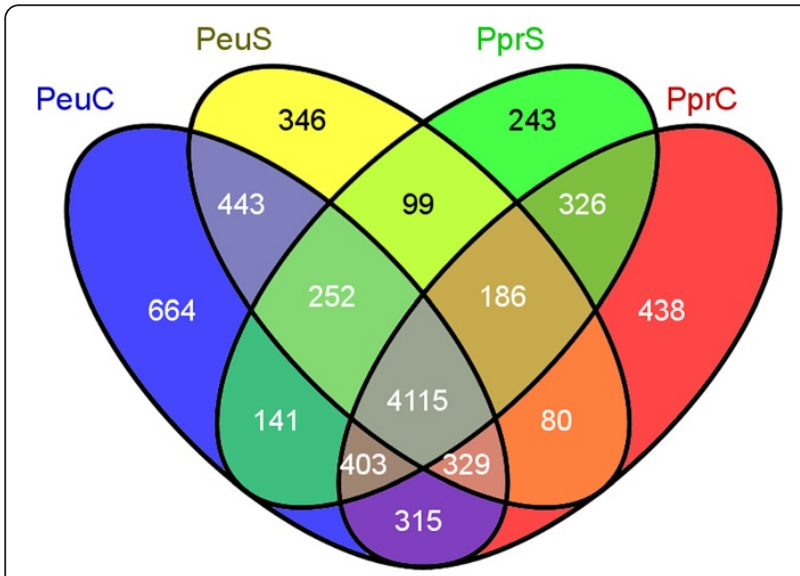

Figure 7 Number of loci showing AS events in $P$. euphratica and $\boldsymbol{P}$. pruinosa. The numbers of loci undergoing AS events in each species and treatment are shown. PeuC, $P$. euphratica control callus; PeuS, P. euphratica salt-stressed callus; PprC, P. pruinosa control callus; PprS, P. pruinosa salt-stressed callus. speciation in their different salty desert habitats. The results of our comparative analyses imply that different species, even sister species, may employ different genetic pathways to cope with salt stress. This suggests that it may be more difficult than previously anticipated to design salt-tolerant plant cultivars $[69,70]$. In order to develop cultivars with high salt tolerance, particular attention should be paid to those genes that are differentially expressed in two or more different species under salt stress. Such genes can be used to facilitate genetic improvement of crops, including cultivated poplars, for growth on saline soils.

\section{Methods}

\section{Gene expression data}

Paired-end RNA-seq reads for control callus and saltstressed callus of $P$. euphratica and $P$. pruinosa, which were obtained by Qiu et al. [27] and Zhang et al. [51], respectively, were downloaded from the NCBI sequence read archive (accession numbers SRX025571, SRX025568, SRX245887 and SRX245885).

We cultured P. euphratica and P. pruinosa calli induced from the shoot under the same conditions. We then replaced the growth medium for one set with the fresh medium and the same medium but supplemented with $100 \mathrm{mM} \mathrm{NaCl}$ (salt stress) for another set. We harvested both sets of calli $24 \mathrm{~h}$ later. The calli from $P$. euphratica and P. pruinosa had the same subculture generation and time and they were highly comparable in terms of physiological state. After RNA extraction and quality determination, we constructed the paired-end cDNA libraries with insert sizes of 200 base pair (bp), and then sequenced the cDNA using an Illumina (San Diego, CA, USA) Genome Analyzer platform according to the manufacturer's protocols with a read length of 75 bp in two lanes. Image output data from the sequencer was transformed into raw sequence data by base calling.

Raw reads generated by Illumina Genome Analyzer were initially processed to obtain clean reads. We first cleaned raw sequence reads by removing exact duplicates from both sequencing directions. We further cleaned reads by removing adapter sequences as well as reads with too many $(>8)$ unknown base calls $(\mathrm{N})$, low complexity, and low-quality bases ( $>50 \%$ of the bases with a quality score $\leq 5$ ). Cleaned reads from each library were used for later differential expression analysis in this study.

\section{Initial mapping of reads}

To determine the level of gene expression, Bowtie2 [71] was used to align RNA-seq reads from the control and salt-stressed samples to transcript sequences from Populus trichocarpa Torr. \& A. Gray [41], using annotation files downloaded from http://www.phytozome.net/poplar (JGI Populus trichocarpa v2.2). No more than a 1 bp 
mismatch was allowed when taking into account differences between the two species. Reads that mapped to reference sequences from multiple genes were filtered out. The remaining clean reads, which were considered to be distinct, were used for further analysis. Transcript abundances were calculated using eXpress [72], which outputs read counts and the number of fragments per kilobase of exon per million fragments mapped (FPKM) [73]. Transcripts with FPKM values $<1$ in both libraries were filtered out and not subjected to further analysis.

\section{Identification of differentially expressed genes}

To identify differentially expressed genes (DEGs) in control callus and salt-stressed callus from $P$. euphratica and $P$. pruinosa, we applied four independent, widely used tools: Cuffdiff [73], DESeq [74], edgeR [75], and EBSeq [76]. Cuffdiff takes a nonparametric, annotation-guided approach to estimating the means and variances of transcript FPKM values under different conditions, using Student's $t$-tests to identify differentially expressed transcripts [73]. In contrast, DESeq, edgeR and EBSeq estimate the means and variances of raw read counts under a negative binomial distribution and use exact tests to identify differentially expressed transcripts. The main difference between DESeq, edgeR and EBSeq is that they use different statistical approaches to estimate variance [74-76]. After the $p$-values for each expressed genes were obtained by the four tools, the false discovery rate (FDR) was used to justify the $p$-value by the function p.adjust in R. Sequences were deemed to be differentially expressed if $\log _{2}$ $\left(\mathrm{FPKM}_{\text {salt }} / \mathrm{FPKM}_{\text {control }}\right)>1$ or $<-1$, and the adjusted $p$-value (FDR) was $<0.05$ as identified by all four metrics.

\section{Functional annotation through BLAST2GO and KEGG}

Gene Ontology (GO) terms were assigned to the identified genes by the blast2GO pipeline [77] using NCBI databases, followed by functional classification using the WEGO software package [78]. For the comparative analysis of DEGs between $P$. euphratica and $P$. pruinosa in response to salinity, singular enrichment analysis (SEA) and parametric analysis of gene set enrichment (PAGE) were performed using the agriGO program (http://bioinfo.cau. edu.cn/agriGO) [79] with the default parameters, using the P. trichocarpa gene models as background, followed by multiple testing with Bonferroni correction (corrected P-value < 0.05). PermutMatrix (Version 1.9.3; http://www. lirmm.fr/ caraux/PermutMatrix/index.html) was used to cluster genes related to plant hormone biosynthesis according to their mean normalized intensity values [80].

\section{Validation of DEG Expression with Quantitative Real-time PCR (qRT-PCR)}

In order to validate the reliability of RNA-Seq experiments, a total of 21 candidate DEGs highly related to salt stress were selected for qRT-PCR test. These genes were chosen for the qRT-PCR analysis based on two criteria: (i) gene's expression patterns between these two species under salt stress should be similar; (ii) it should have only one BLAST hit when searching against genes of Arabidopsis thaliana to exclude paralogs. A total of $0.5 \mu \mathrm{g}$ of DNase I-treated total RNA was converted into single-stranded cDNA using a Prime-Script 1st Strand cDNA Synthesis Kit (TaKaRa, Dalian, China). The cDNA templates were then diluted 20-fold before use. The quantitative reaction was performed on a CFX96 RealTime PCR Detection System (Bio-Rad, Singapore) using SYBR Premix Ex Taq ${ }^{\text {sax }}$ (TaKaRa, Dalian, China). PCR amplification was performed under the following conditions: $30 \mathrm{~s}$ at $95^{\circ} \mathrm{C}$, followed by 40 cycles of $95^{\circ} \mathrm{C}$ for $15 \mathrm{~s}, 60^{\circ} \mathrm{C}$ for $30 \mathrm{~s}$ and then $72^{\circ} \mathrm{C}$ for $20 \mathrm{~s}$. All primers were designed using PRIMER3 software and were listed in Additional file 8. Three biological replicates based the calli cultured from different individuals with the same subculture and physiological state were performed in order to exclude sampling errors. The relative expression levels of the selected DEGs normalized to an internal reference gene actin was calculated using $2^{-\Delta \Delta C t}$ method [81].

\section{Identification of alternative splicing}

We prepared a database of all possible splice junctions between annotated exons in each selected gene and identified new possible junctions using TopHat [82]. We combined these two databases, removing any redundancy between them, and then extracted junction sequences of width 65 bases on each side from all the above junctions. To evaluate which of these junctions were validated by our Illumina reads, we aligned reads from each library separately against the junction sequences, allowing up to one mismatch (in a read of $75 \mathrm{bp}$ ). If at least two reads aligned to a splice junction, we considered it to be validated.

Six different types of alternative mRNA processing events were analysed [83]. We first considered skipped exons (SE), in which one or more exons are spliced out of the mature message, and retained introns (RI), in which one or more introns are included in the message. Also included were alternative 5' splice site (A5SS) and alternative 3' splice site (A3SS) events, which are particularly difficult to interrogate by microarray analysis because the variably included region is often quite small. Finally, alternative last exons (ALEs) in which alternative use of a pair of polyadenylation sites results in distinct terminal exons, and alternative first exons (AFEs), where alternative promoter use results in mRNA isoforms with distinct 5' UTRs, were considered. 


\section{Additional files}

\begin{abstract}
Additional file 1: Eight clusters of DEGs under salt stress.
Additional file 2: $\mathrm{GO}$ enrichment analyses for the eight clusters of DEGs under salt stress. GO terms, identified by SEA analysis as significant, for co-regulated and species-specific DEGs up- or down-regulated in P. pruinosa and $P$. euphratica under salinity stress. The categories 'down-regulated in P. pruinosa and up-regulated in P. euphratica' and 'up-regulated in P. pruinosa and down-regulated in $P$. euphratica' were excluded because only one gene fell into each of these categories.
\end{abstract}

Additional file 3: Genes involved in the biosynthesis of plant hormones. Additional file 4: Ninety-nine DEGs were categorized as transporters. Additional file 5: Transcription factors in Populus trichocarpa.

Additional file 6: One hundred and fifteen DEGs were categorized as transcription factors. Each transcription factor contains known DNA-binding domains defined in the Pfam database.

Additional file 7: GO enrichment analyses for the 346 and 243 loci showing AS events solely in either $P$. euphratica or $P$. pruinosa in response to salt stress.

Additional file 8: Primer used for real-time quantitative RT-PCR in this study.

\section{Abbreviations}

DGE: Digital gene expression; GO: Gene ontology; KEGG: Kyoto Encyclopedia of Genes and Genomes; FPKM: Number of fragments per kilobase of exon per million fragments mapped; DEGs: Differentially expressed genes; PAGE: Parametric analysis of gene set enrichment; SEA: Singular enrichment analysis; FDR: False discovery rate; qRT-PCR: Quantitative real-time polymerase chain reaction.

\section{Competing interests}

The authors declare that they have no competing interests.

\section{Authors' contributions}

$J L$ : conceived and designed the project. JZ: performed the experiments, analyzed the data and wrote the manuscript. JF: performed the experiments and downstream bioinformatics analyses. JL: performed the qRT-PCR experiments. YY: performed alternative splicing analyses. XZ: performed the qRT-PCR experiments. DW: critically reviewed the manuscript and was the main advisor for the experimental plan. All authors read and approved the final manuscript.

\section{Acknowledgements}

Financial support was provided by the National High-Tech Research and Development Program of China (863 Program, No. 2013AA102605), the National Key Project for Basic Research (2012CB114504), the National Science foundation of China (30972336, 31270652), and the Xinjiang Production \& Construction Corps Key Laboratory of Protection and Utilization of Biological Resources in Tarim Basin opening topic fund (BRZD1204). The funders had no role in the study design, data collection and analysis, decision to publish, or preparation of the manuscript.

\section{Author details}

'State Key Laboratory of Grassland and Agro-Ecosystems, School of Life Sciences, Lanzhou University, Lanzhou 730000, Gansu, China. ${ }^{2}$ Xinjiang Production \& Construction Corps Key Laboratory of Protection and Utilization of Biological Resources in Tarim Basin, College of Plant Science, Tarim University, Alar 843300, Xinjiang, China.

Received: 13 July 2013 Accepted: 30 April 2014

Published: 4 May 2014

\section{References}

1. Boyer JS: Plant productivity and environment. Science 1982, 218(4571):443-448.

2. Rengasamy P: World salinization with emphasis on Australia. J Exp Bot 2006, 57(5):1017-1023.

3. Munns R, Tester M: Mechanisms of salinity tolerance. Annu Rev Plant Biol 2008, 59(1):651-681.
4. Langridge P, Paltridge $N$, Fincher $G$ : Functional genomics of abiotic stress tolerance in cereals. Briefings Funct Genomics Proteomics 2006, 4(4):343-354.

5. Bohnert $H J$, Nelson DE, Jensen RG: Adaptations to environmental stresses. Plant Cell Online 1995, 7(7):1099-1111.

6. Bartels D, Sunkar R: Drought and salt tolerance in plants. Crit Rev Plant Sci 2005, 24(1):23-58.

7. Ma Q, Dai X, Xu Y, Guo J, Liu Y, Chen N, Xiao J, Zhang D, Xu Z, Zhang X, Chong K: Enhanced tolerance to chilling stress in OSMYB3R-2 transgenic rice is mediated by alteration in cell cycle and ectopic expression of stress genes. Plant Physiol 2009, 150(1):244-256.

8. Xu Z-S, Xia L-Q, Chen M, Cheng X-G, Zhang R-Y, Li L-C, Zhao Y-X, Lu Y, Ni Z-Y, Liu L, Qiu ZG, Ma YZ: Isolation and molecular characterization of the Triticum aestivum L. ethylene-responsive factor 1 (TaERF1) that increases multiple stress tolerance. Plant Mol Biol 2007, 65(6):719-732.

9. Urano K, Kurihara Y, Seki M, Shinozaki K: 'Omics' analyses of regulatory networks in plant abiotic stress responses. Curr Opin Plant Biol 2010, 13(2):132-138.

10. Close TJ: Dehydrins: Emergence of a biochemical role of a family of plant dehydration proteins. Physiol Plant 1996, 97(4):795-803.

11. Forrest $\mathrm{K}$, Bhave M: Major intrinsic proteins (MIPs) in plants: a complex gene family with major impacts on plant phenotype. Funct Integr Genomics 2007, 7(4):263-289.

12. Meiri D, Breiman A: Arabidopsis ROF1 (FKBP62) modulates thermotolerance by interacting with HSP90.1 and affecting the accumulation of HsfA2-regulated sHSPs. The. Plant J 2009, 59(3):387-399.

13. Munns R: Genes and salt tolerance: bringing them together. New Phytol 2005, 167(3):645-663.

14. Selote DS, Khanna-Chopra R: Drought acclimation confers oxidative stress tolerance by inducing co-ordinated antioxidant defense at cellular and subcellular level in leaves of wheat seedlings. Physiol Plant 2006, 127(3):494-506.

15. Tyerman SD, Niemietz CM, Bramley H: Plant aquaporins: multifunctional water and solute channels with expanding roles. Plant Cell Environ 2002, 25(2):173-194.

16. Hasegawa PM, Bressan RA, Zhu J-K, Bohnert HJ: Plant cellular and molecular responses to high salinity. Annu Rev Plant Physiol Plant Mol Biol 2000, 51(1):463-499.

17. Seki M, Narusaka M, Abe H, Kasuga M, Yamaguchi-Shinozaki K, Carninci P, Hayashizaki Y, Shinozaki K: Monitoring the Expression Pattern of 1300 Arabidopsis Genes under Drought and Cold Stresses by Using a Full-Length cDNA Microarray. Plant Cell Online 2001, 13(1):61-72.

18. Yamaguchi-Shinozaki K, Shinozaki K: Transcriptional regulatory networks in cellular responses and tolerance to dehydration and cold stresses. Annu Rev Plant Biol 2006, 57(1):781-803.

19. Deyholos MK: Making the most of drought and salinity transcriptomics. Plant Cell Environ 2010, 33(4):648-654.

20. Gong Q, Li P, Ma S, Indu Rupassara S, Bohnert HJ: Salinity stress adaptation competence in the extremophile Thellungiella halophila in comparison with its relative Arabidopsis thaliana. Plant J 2005, 44(5):826-839.

21. Taji T, Seki M, Satou M, Sakurai T, Kobayashi M, Ishiyama K, Narusaka Y, Narusaka M, Zhu J-K, Shinozaki K: Comparative Genomics in Salt Tolerance between Arabidopsis and Arabidopsis-Related Halophyte Salt Cress Using Arabidopsis Microarray. Plant Physiol 2004, 135(3):1697-1709.

22. Wong CE, Li Y, Labbe A, Guevara D, Nuin P, Whitty B, Diaz C, Golding GB, Gray GR, Weretilnyk EA, Griffith M, Moffatt BA: Transcriptional Profiling Implicates Novel Interactions between Abiotic Stress and Hormonal Responses in Thellungiella, a Close Relative of Arabidopsis. Plant Physiol 2006, 140(4):1437-1450.

23. Rabello A, Guimaraes C, Rangel P, Da Silva F, Seixas D, De Souza E, Brasileiro A, Spehar C, Ferreira M, Mehta A: Identification of drought-responsive genes in roots of upland rice (Oryza sativa L). BMC Genomics 2008, 9(1):485.

24. Beritognolo I, Harfouche A, Brilli F, Prosperini G, Gaudet M, Brosche M, Salani F, Kuzminsky E, Auvinen P, Paulin L, Kangasjärvi J, Loreto F, Valentini R, Mugnozza GS, Sabatti M: Comparative study of transcriptional and physiological responses to salinity stress in two contrasting Populus alba L. genotypes. Tree Physiol 2011, 31(12):1335-1355.

25. Cohen D, Bogeat-Triboulot M-B, Tisserant E, Balzergue S, Martin-Magniette M-L, Lelandais G, Ningre N, Renou J-P, Tamby J-P, Le Thiec D, Hummel I: Comparative transcriptomics of drought responses in Populus: a metaanalysis of genome-wide expression profiling in mature leaves and root apices across two genotypes. BMC Genomics 2010, 11(1):630. 
26. Janz D, Behnke K, Schnitzler JP, Kanawati B, Schmitt-Kopplin P, Polle A: Pathway analysis of the transcriptome and metabolome of salt sensitive and tolerant poplar species reveals evolutionary adaption of stress tolerance mechanisms. BMC Plant Biol 2010, 10:150.

27. Qiu Q, Ma T, Hu Q, Liu B, Wu Y, Zhou H, Wang Q, Wang J, Liu J: Genome-scale transcriptome analysis of the desert poplar, Populus euphratica. Tree Physiol 2011, 31(4):452-461.

28. Sun W, Xu X, Zhu H, Liu A, Liu L, Li J, Hua X: Comparative transcriptomic profiling of a salt-tolerant wild tomato species and a salt-sensitive tomato cultivar. Plant Cell physiol 2010, 51(6):997-1006.

29. Mane SP, Robinet CV, Ulanov A, Schafleitner R, Tincopa L, Gaudin A, Nomberto G, Alvarado C, Solis C, Bolivar LA, Blas R, Ortega O, Solis J, Panta A, Rivera C, Samolski I, Carbajulca DH, Bonierbale M, Pati A, Heath LS, Bohnert HJ, Grene R: Molecular and physiological adaptation to prolonged drought stress in the leaves of two Andean potato genotypes. Funct Plant Biol 2008, 35(8):669-688.

30. Zahaf O, Blanchet S, De Zélicourt A, Alunni B, Plet J, Laffont C, De Lorenzo L, Imbeaud S, Ichanté J-L, Diet A, Badri M, Zabalza A, González EM, Delacroix H, Gruber V, Frugier F, Crespi M: Comparative Transcriptomic Analysis of Salt Adaptation in Roots of Contrasting Medicago truncatula Genotypes. Mol Plant 2012, 5(5):1068-1081.

31. Rodrigues FA, De Laia ML, Zingaretti SM: Analysis of gene expression profiles under water stress in tolerant and sensitive sugarcane plants. Plant Sci 2009, 176(2):286-302.

32. Bazakos C, Manioudaki ME, Therios I, Voyiatzis D, Kafetzopoulos D, Awada T, Kalaitzis P: Comparative transcriptome analysis of two olive cultivars in response to $\mathrm{NaCl}$-stress. PLoS One 2012, 7(8):e42931.

33. Chen W, Provart NJ, Glazebrook J, Katagiri F, Chang H-S, Eulgem T, Mauch F, Luan S, Zou G, Whitham SA, Budworth PR, Tao Y, Xie Z, Chen X, Lam S, Kreps JA, Harper JF, Si-Ammour A, Mauch-Mani B, Heinlein M, Kobayashi K, Hohn T, Dangl JL, Wang X, Zhu T: Expression Profile Matrix of Arabidopsis Transcription Factor Genes Suggests Their Putative Functions in Response to Environmental Stresses. Plant Cell Online 2002, 14(3):559-574.

34. He $X$, Hou $X$, Shen $Y$, Huang Z: TaSRG, a wheat transcription factor, significantly affects salt tolerance in transgenic rice and Arabidopsis. FEBS Lett 2011, 585(8):1231-1237.

35. Ingram J, Bartels D: The molecular basis of dehydration tolerance in plants. Annu Rev Plant Physiol Plant Mol Biol 1996, 47(1):377-403.

36. Seki M, Narusaka M, Ishida J, Nanjo T, Fujita M, Oono Y, Kamiya A, Nakajima M, Enju A, Sakurai T, Satou M, Akiyama K, Taji T, Yamaguchi-Shinozaki K, Carninci P, Kawai J, Hayashizaki Y, Shinozaki K: Monitoring the expression profiles of 7000 Arabidopsis genes under drought, cold and high-salinity stresses using a full-length cDNA microarray. Plant J 2002, 31(3):279-292.

37. Shinozaki K, Yamaguchi-Shinozaki K, Seki M: Regulatory network of gene expression in the drought and cold stress responses. Curr Opin Plant Biol 2003, 6(5):410-417

38. Xiong L, Schumaker KS, Zhu J-K: Cell Signaling during Cold, Drought, and Salt Stress. Plant Cell Online 2002, 14(suppl 1):S165-S183.

39. Zhu J-K: Salt and drought stress signal transduction in plants. Annu Rev Plant Biol 2002, 53(1):247-273.

40. Chen S, Polle A: Salinity tolerance of Populus. Plant Biol 2010, 12(2):317-333.

41. Tuskan GA, DiFazio S, Jansson S, Bohlmann J, Grigoriev I, Hellsten U, Putnam N, Ralph S, Rombauts S, Salamov A, Schein J, Sterck L, Aerts A, Bhalerao RR, Bhalerao RP, Blaudez D, Boerjan W, Brun A, Brunner A, Busov V, Campbell M, Carlson J, Chalot M, Chapman J, Chen GL, Cooper D, Coutinho PM, Couturier J, Covert S, Cronk Q, et al: The Genome of Black Cottonwood, Populus trichocarpa (Torr. \& Gray). Science 2006, 313(5793):1596-1604.

42. Wullschleger SD, Weston DJ, DiFazio SP, Tuskan GA: Revisiting the sequencing of the first tree genome: Populus trichocarpa. Tree Physiol 2013, 33(4):357-364.

43. Eckenwalder JE: Systematics and evolution of Populus. In In Biology of Populus. Edited by Stettler RF, Heilman JPE, Hinckley TM. Ottawa: NRC Research Press; 1996:7-32.

44. Hukin D, Cochard H, Dreyer E, Thiec DL, Bogeat-Triboulot MB: Cavitation vulnerability in roots and shoots: does Populus euphratica Oliv., a poplar from arid areas of Central Asia, differ from other poplar species? J Exp Bot 2005, 56(418):2003-2010.

45. Ma H-C, Fung L, Wang S-S, Altman A, Hüttermann A: Photosynthetic response of Populus euphratica to salt stress. For Ecol Manag 1997 93(1-2):55-61.
46. Wang J, Wu Y, Ren G, Guo Q, Liu J, Lascoux M: Genetic Differentiation and Delimitation between Ecologically Diverged Populus euphratica and P. pruinosa. PLoS One 2011, 6(10):e26530.

47. Chen S, Li J, Fritz E, Wang S, Hüttermann A: Sodium and chloride distribution in roots and transport in three poplar genotypes under increasing $\mathrm{NaCl}$ stress. For Ecol Manag 2002, 168(1-3):217-230.

48. Ottow EA, Brinker $M$, Teichmann T, Fritz E, Kaiser W, Brosché M, Kangasjärvi J, Jiang X, Polle A: Populus euphratica Displays Apoplastic Sodium Accumulation, Osmotic Adjustment by Decreases in Calcium and Soluble Carbohydrates, and Develops Leaf Succulence under Salt Stress. Plant Physiol 2005, 139(4):1762-1772.

49. Wu Y, Ding N, Zhao X, Zhao M, Chang Z, Liu J, Zhang L: Molecular characterization of PeSOS1: the putative $\mathrm{Na}+/ \mathrm{H}+$ antiporter of Populus euphratica. Plant Mol Biol 2007, 65(1-2):1-11.

50. Zhang F, Wang Y, Yang Y, Wu HAO, Wang DI, Liu J: Involvement of hydrogen peroxide and nitric oxide in salt resistance in the calluses from Populus euphratica. Plant Cell Environ 2007, 30(7):775-785.

51. Zhang J, Xie P, Lascoux M, Meagher TR, Liu J: Rapidly Evolving Genes and Stress Adaptation of Two Desert Poplars, Populus euphratica and P. pruinosa. PLoS One 2013, 8(6):e66370.

52. Peng S, Jiang H, Zhang S, Chen L, Li X, Korpelainen H, Li C: Transcriptional profiling reveals sexual differences of the leaf transcriptomes in response to drought stress in Populus yunnanensis. Tree Physiol 2012, 32(12):1541-1555.

53. Sun J, Chen S, Dai S, Wang R, Li N, Shen X, Zhou X, Lu C, Zheng X, Hu Z, Zhang Z, Song J, Xu Y: NaCl-Induced Alternations of Cellular and Tissue Ion Fluxes in Roots of Salt-Resistant and Salt-Sensitive Poplar Species. Plant Physio/ 2009, 149(2):1141-1153.

54. Wang R, Chen S, Zhou X, Shen X, Deng L, Zhu H, Shao J, Shi Y, Dai S, Fritz E, Hüttermann A, Polle A: Ionic homeostasis and reactive oxygen species control in leaves and xylem sap of two poplars subjected to $\mathrm{NaCl}$ stress. Tree Physiol 2008, 28(6):947-957.

55. Ye C-Y, Zhang H-C, Chen J-H, Xia X-L, Yin W-L: Molecular characterization of putative vacuolar $\mathrm{NHX}$-type $\mathrm{Na}+/ \mathrm{H}+$ exchanger genes from the salt-resistant tree Populus euphratica. Physiol Plant 2009, 137(2):166-174.

56. Mizoi J, Shinozaki K, Yamaguchi-Shinozaki K: AP2/ERF family transcription factors in plant abiotic stress responses. Biochim Biophys Acta-Gene Regul Mech 2012, 1819(2):86-96.

57. Oh S-J, Song SI, Kim YS, Jang H-J, Kim SY, Kim M, Kim Y-K, Nahm BH, Kim J-K: Arabidopsis CBF3/DREB1A and ABF3 in Transgenic Rice Increased Tolerance to Abiotic Stress without Stunting Growth. Plant Physiol 2005, 138(1):341-351.

58. Kumar GR, Sakthivel K, Sundaram RM, Neeraja CN, Balachandran SM, Rani NS, Viraktamath BC, Madhav MS: Allele mining in crops: Prospects and potentials. Biotechnol Adv 2010, 28(4):451-461.

59. Latha R, Rubia L, Bennett J, Swaminathan MS: Allele mining for stress tolerance genes in Oryza species and related germplasm. Mol Biotechnol 2004, 27(2):101-108.

60. Jiang S-Y, Ma A, Ramamoorthy R, Ramachandran S: Genome-Wide Survey on Genomic Variation, Expression Divergence, and Evolution in Two Contrasting Rice Genotypes under High Salinity Stress. Genome Biol Evol 2013, 5(11):2032-2050.

61. Taji T, Ohsumi C, luchi S, Seki M, Kasuga M, Kobayashi M, Yamaguchi-Shinozaki K, Shinozaki K: Important roles of drought- and cold-inducible genes for galactinol synthase in stress tolerance in Arabidopsis thaliana. Plant Journal 2002, 29(4):417-426.

62. Panikulangara TJ, Eggers-Schumacher G, Wunderlich M, Stransky H, Schöffl F: Galactinol synthase1. A Novel Heat Shock Factor Target Gene Responsible for Heat-Induced Synthesis of Raffinose Family Oligosaccharides in Arabidopsis. Plant physiology 2004, 136(2):3148-3158.

63. Gu R, Fonseca S, Puskás LG, Hackler L, Zvara Á, Dudits D, Pais MS: Transcript identification and profiling during salt stress and recovery of Populus euphratica. Tree Physiol 2004, 24(3):265-276.

64. Li B, Duan H, Li J, Deng X, Yin W, Xia X: Global identification of miRNAs and targets in Populus euphratica under salt stress. Plant Mol Biol 2013, 81(6):525-539.

65. Chaves MM, Flexas J, Pinheiro C: Photosynthesis under drought and salt stress: regulation mechanisms from whole plant to cell. Ann Bot 2009, 103(4):551-560.

66. Bogeat-Triboulot M-B, Brosché M, Renaut J, Jouve L, Le Thiec D, Fayyaz $P$, Vinocur B, Witters E, Laukens K, Teichmann T, Altman A, Hausman JF, Polle 
A, Kangasjärvi J, Dreyer E: Gradual Soil Water Depletion Results in Reversible Changes of Gene Expression, Protein Profiles, Ecophysiology, and Growth Performance in Populus euphratica, a Poplar Growing in Arid Regions. Plant Physiol 2007, 143(2):876-892.

67. Tang S, Liang H, Yan D, Zhao Y, Han X, Carlson J, Xia X, Yin W: Populus euphratica: the transcriptomic response to drought stress. Plant Mol Biol 2013, 83(6):539-557.

68. Wang B-B, Brendel V: Genomewide comparative analysis of alternative splicing in plants. Proc Natl Acad Sci 2006, 103(18):7175-7180.

69. Polle A, Douglas $C$ : The molecular physiology of poplars: paving the way for knowledge-based biomass production. Plant Biol 2010, 12(2):239-241.

70. Postnikova OA, Shao J, Nemchinov LG: Analysis of the alfalfa root transcriptome in response to salinity stress. Plant \& cell physiology 2013. 54(7):1041-1055.

71. Langmead B, Trapnell C, Pop M, Salzberg S: Ultrafast and memory-efficient alignment of short DNA sequences to the human genome. Genome Biol 2009, 10(3):R25.

72. Roberts $A$, Pachter $L$ : Streaming fragment assignment for real-time analysis of sequencing experiments. Nat Meth 2013, 10(1):71-73.

73. Trapnell C, Williams BA, Pertea G, Mortazavi A, Kwan G, Van Baren MJ, Salzberg SL, Wold BJ, Pachter L: Transcript assembly and quantification by RNA-Seq reveals unannotated transcripts and isoform switching during cell differentiation. Nat Biotech 2010, 28(5):511-515.

74. Anders S, Huber W: Differential expression analysis for sequence count data. Genome Biol 2010, 11(10):R106.

75. Robinson MD, McCarthy DJ, Smyth GK: edgeR: a Bioconductor package for differential expression analysis of digital gene expression data. Bioinformatics 2010, 26(1):139-140.

76. Leng N, Dawson JA, Thomson JA, Ruotti V, Rissman Al, Smits BMG, Haag JD, Gould MN, Stewart RM, Kendziorski C: EBSeq: an empirical Bayes hierarchical model for inference in RNA-seq experiments. Bioinformatics 2013, 29(8):1035-1043.

77. Conesa A, Götz S, García-Gómez JM, Terol J, Talón M, Robles M: Blast2GO: a universal tool for annotation, visualization and analysis in functional genomics research. Bioinformatics 2005, 21(18):3674-3676.

78. Ye J, Fang L, Zheng H, Zhang Y, Chen J, Zhang Z, Wang J, Li S, Li R, Bolund L, Wang J: WEGO: a web tool for plotting GO annotations. Nucleic Acids Res 2006, 34(suppl 2):W293-W297.

79. Du Z, Zhou X, Ling Y, Zhang Z, Su Z: agriGO: a GO analysis toolkit for the agricultural community. Nucleic Acids Res 2010, 38(suppl 2):W64-W70

80. Zhang S, Chen F, Peng S, Ma W, Korpelainen H, Li C: Comparative physiological, ultrastructural and proteomic analyses reveal sexual differences in the responses of Populus cathayana under drought stress. Proteomics 2010, 10(14):2661-2677.

81. Livak KJ, Schmittgen TD: Analysis of Relative Gene Expression Data Using Real-Time Quantitative PCR and the $2^{-\Delta \Delta C T}$ Method. Methods 2001, 25(4):402-408.

82. Trapnell C, Pachter L, Salzberg SL: TopHat: discovering splice junctions with RNA-Seq. Bioinformatics 2009, 25(9):1105-1111.

83. Wang ET, Sandberg R, Luo S, Khrebtukova I, Zhang L, Mayr C, Kingsmore SF, Schroth GP, Burge CB: Alternative isoform regulation in human tissue transcriptomes. Nature 2008, 456(7221):470-476.

doi:10.1186/1471-2164-15-337

Cite this article as: Zhang et al:: Transcriptome differences between two sister desert poplar species under salt stress. BMC Genomics 2014 15:337.

\section{Submit your next manuscript to BioMed Central and take full advantage of:}

- Convenient online submission

- Thorough peer review

- No space constraints or color figure charges

- Immediate publication on acceptance

- Inclusion in PubMed, CAS, Scopus and Google Scholar

- Research which is freely available for redistribution

Submit your manuscript at www.biomedcentral.com/submit 